This item was submitted to Loughborough's Research Repository by the author.

Items in Figshare are protected by copyright, with all rights reserved, unless otherwise indicated.

\title{
Fractal structures in systems made of small magnetic particles
}

PLEASE CITE THE PUBLISHED VERSION

PUBLISHER

(C) American Physical Society

LICENCE

CC BY-NC-ND 4.0

\section{REPOSITORY RECORD}

Kuerten, K.E., and F.V. Kusmartsev. 2019. "Fractal Structures in Systems Made of Small Magnetic Particles". figshare. https://hdl.handle.net/2134/1309. 


\title{
Fractal structures in systems made of small magnetic particles
}

\author{
Karl E. Kürten ${ }^{1}$ and Feodor V. Kusmartsev ${ }^{2}$ \\ ${ }^{1}$ Institut für Experimentalphysik, Universität Wien, Austria \\ ${ }^{2}$ Department of Physics, Loughborough University, Loughborough LE11 3TU, United Kingdom \\ (Received 29 December 2004; revised manuscript received 14 March 2005; published 14 July 2005)
}

\begin{abstract}
We have found that in a system consisting of small magnetic particles a phenomenon related to the formation of fractal structures may arise. The fractal features may arise not only in the distribution of magnetic moments but also in their energy spectrum. The magnetization and the susceptibility of the system also display fractal characteristics. The multiple structures are associated with exponentially many locally stable minima in a highly complex energy landscape. The signature of these fractal structures can be experimentally detected by various methods.
\end{abstract}

DOI: 10.1103/PhysRevB.72.014433

PACS number(s): 75.75.+a, 75.60.Jk, 75.50.Tt, 75.10.Hk

\section{INTRODUCTION}

Modern technologies, such as molecular beam epitaxy and laser ablation, open the way to grow physical systems with properties of technological interest and an enormous scope of useful applications. ${ }^{1}$ Functionalizing of the individual nanoclusters is part of the next big push in nanotechnology. Therefore magnetic nanoparticles and different structures made of these nanoparticles have been the focus of intense research for the last decade. ${ }^{2}$ There is also a growing realization of the enormous potential of cluster assembled films and other nanostructures in the production of high performance materials. ${ }^{3}$ Transmission electron microscopy (TEM) allows one to investigate and control the magnetic properties of artificial structures, films, and multilayers made of such small particles. These magnetic microstructures evolve with magnetic field and temperature and display many unusual features. ${ }^{4}$ The control and understanding of these structures will give advances in applications especially related to formation of spintronic circuits operating in the gigahertz $(\mathrm{GHz})$ and terahertz $(\mathrm{THz})$ range as well as many other properties related to the formation of magnetic memory used in hard disks. Among the most extensively studied systems are arrays formed from small magnetic particles. ${ }^{1,5-7}$ For example, the control of magnetization reversal involving well-defined domain states in nanomagnet arrays is a key to future applications for magnetic recording and magnetoelectronic devices. ${ }^{8-10}$ Arrays formed from nanoparticles may also have very complex behavior of magnetization which could be associated with complex magnetic domain structures. A simple scheme to extract the magnetization reversal of characteristic domains on nanoparticle arrays from soft X-ray has been recently demonstrated. ${ }^{11}$ A control of the magnetization reversal is based on the knowledge about the reproducible domain structures.

For a macroscopic sample the formation of domains is not controllable. The situation changes when the size of the sample decreases. A very small ferromagnetic nanoparticle of a few nanometers size is typically domain free. When the size increases a few controllable domains may appear inside the nanoparticle. For this purpose, in order to control the formation of domains in small microscopic systems such as nanometers disks ${ }^{12}$ and other complicated rings ${ }^{13}$ small me- soscopic systems have been thoroughly investigated.

However, there is another way to get controllable domain structures by assembly of similar or different mesoscopic samples from domain-free small nanoparticles. In this case any interaction between nanoparticles is much smaller than the exchange interaction between microscopic moments within each single small nanoparticle. Due to this fact the energy cost for domain creation within each single nanoparticle is much larger than the energy cost for the domain formation between the nanoparticles. Therefore for a system consisting of very small magnetic nanoparticles domains are formed mostly in the boundary areas between nanoparticles.

For example, a typical such system consists of nanoparticles with size smaller than $100 \mathrm{~nm}$ made of "supermalloy" $\mathrm{Ni}_{80} \mathrm{Fe}_{14} \mathrm{Mo}_{5}$. Each of the particles is a single domain object. ${ }^{14}$ These particles also have an unique property related to a configurational anisotropy, which, in turn, is strongly related to the shape of the particles. ${ }^{15}$ For example, for the particles having an ellipsoidal shape the magnetic moment will be directed along the axes of the prolongation.

Recently Cowburn and Welland have proposed using one such system, namely, to use a chain of such magnetic nanoparticles deposited on a nonmagnetic substrate as a room temperature magnetic quantum cellular automaton (MQCA). ${ }^{16}$ They have produced such a chain where all particles were ferromagnetically coupled and oriented along the chain. The orientation of the ferromagnetic moments has been controlled by a deposition of a first particle of the chain which was larger than the other particles and had a distinguished ellipsoidal shape. In that paper ${ }^{16}$ it was shown that by slightly biased, pulsed magnetic field the magnetic moments associated with these individual particles are flipped coherently, comparable to a "domino" effect. Therefore it was concluded that such a chain has all the properties needed to form a quantum cellular automaton.

\section{THE MODEL}

In order to describe the formation of domains and other magnetic structures in microscopic systems made of small magnetic nanoparticles we propose here a theoretical model. In particular, using this model we investigate the formation 
of domain structures in a linear chain and in other small multiparticle clusters. We investigate this model in detail considering arrays of a few and many nanoparticles, and shown that they have a strong potential for sensor applications and data storage. In particular we have shown that such systems have a very complex nontrivial magnetic behavior and therewith different nontrivial structures displaying fractal features may be formed. Specifically the system behavior (with increasing particle number), the values of magnetic moments, the energy spectrum, coercive forces, hysteresis loops, and all such properties may display the features of a fractal. The formation of these fractal structures is mostly related to the discrete nature of the systems made of small particles and does not depend on the specific models which we have been considering. Therefore the phenomenon of the fractal creation has a very general character and must be taken into account in the design of any system made of small particles and having a potential for applications.

For an illustration of the fractal formation in the present work we consider one of these systems made of small particles, namely, a linear chain. Such system can be and has already been produced from small ferromagnetic particles made, for example, of $\mathrm{Fe}$ (see, also, Ref. 16). If all spins within a single particle are ferromagnetically ordered, then each particle may be considered as having a single classical spin $\vec{S}$. The value of $\vec{S}$ may be well described by a GinzburgLandau model applied to the single particle

$$
F_{\text {single }}=\frac{A}{2} \vec{S}_{i}^{2}+\frac{B}{4} \vec{S}_{i}^{4}-\vec{H} \vec{S}_{i}
$$

where $\vec{S}=\left(S_{x}, S_{y}, S_{z}\right)$; here $A=a\left(T-T_{c}\right)$ and $B$ are phenomenological constants and $\vec{H}$ is an external magnetic field. The relative orientation of the total magnetic moment of each single particle is determined by anisotropy constants. The shape of the particles gives rise to the so-called configurational anisotropy, which was not included yet. For small particles the value of the configurational anisotropy is the largest. This situation with the largest configurational anisotropy can be readily produced for all magnetic particles having, for example, a needle shape, so that each individual magnetic moment associated with each single particles can be oriented perpendicular to or along the chain of these particles. ${ }^{15,16}$ Let us assume that all particles have such a shape which gives the strongest configurational anisotropy. For example, suppose all particles have ellipsoidal shape prolongated along the $z$ direction. Then due to the configurational anisotropy all magnetic moments associated with single individual particles are oriented along the $z$ direction. Therefore, since the magnetic moments of all particles will be collinear, we can introduce the ansatz $\vec{S}_{i}=\left(0,0, S z_{i}\right)$, where the magnitude $z_{i}$ describes the value of the magnetic moment in the $i$ th particle. Below we show that this ansatz arises exactly as a result of perturbation theory expansion in the limit of a large anisotropy constant $K$ in a comparison with a interparticle interaction $J$. We assume also that the magnetic field is oriented along the axis of the anisotropy as $\vec{H}=(0,0, H)$ and the configurational anisotropy constant $K$ is much larger than the coupling constant $J$ of the exchange or dipolar interaction between particles, which was probed in experiments by Cowburn. ${ }^{17}$

These assumptions are plausible and will strongly simplify the considered model for a system made of these small particles. This will allow us to find exact analytic nontrivial solutions describing all states, magnetic properties, and energy spectra of the system under consideration. The total distribution of moments between the particles are also determined by an exchange and dipole-dipole interactions between the magnetic particles. The chain of magnetic particles can be described by the Hamiltonian

$F=-J \sum_{\langle i, j\rangle} \vec{S}_{i} \vec{S}_{j}+\frac{A}{2} \sum_{i} \vec{S}_{i}^{2}+\frac{B}{4} \sum_{i} \vec{S}_{i}^{4}-\sum_{i} \vec{H} \vec{S}_{i}+K \sum_{i}\left(S_{i x}^{2}+S_{i y}^{2}\right)$,

where we assume that particles are interacting ferromagnetically with the exchange constant $J$ and the value of the configurational anisotropy constant $K / J \rightarrow \infty$ and $-K / A \rightarrow \infty$. Let us make an expansion of the free energy with respect to these very large parameters. The stationary configurations of the magnetic moments are determined with the use of equations obtained by the minimisation of $F$ with respect to the components $S_{i x}, S_{i y}$, and $S_{i z}$. In zero approximation with respect to the very large parameters, $K / J \gg 1$ and $-K / A \gg 1$ the equations obtained for the first two components have a very simple form: $S_{i x}=0$ and $S_{i y}=0$ and obvious solutions. Taking into account these solutions, the form of the vectors describing the magnetic moments, $\vec{S}_{i}$, is simplified to the form $\vec{S}_{i}$ $=\left(0,0, S z_{i}\right)$. After the substitution of this form for $\vec{S}_{i}$ the terms with large parameters in the expression for the free energy $F$ disappear and it is simplified to the form

$$
\begin{aligned}
F= & \frac{J S^{2}}{2} \sum_{\langle i, j\rangle}\left(z_{i}-z_{j}\right)^{2}+\left(\frac{A}{2} S^{2}-J S^{2}\right) \sum_{i} z_{i}^{2}+\frac{B}{4} S^{4} \sum_{i} z_{i}^{4} \\
& -H S \sum_{i} z_{i} .
\end{aligned}
$$

Let us make the substitution $z_{i}=\sqrt{\left(\alpha J / B S^{2}\right)} x_{i}$. After this substitution the expression for the free energy, Eq. (3) will be transformed into a new form. Then, after rescaling and using dimensionless units we obtain a standard model as

$$
\mathbf{F}\left(x_{1}, x_{2}, \ldots, x_{N}\right)=\sum_{i=1}^{N}\left[\frac{1}{2}\left(x_{i}-x_{i-1}\right)^{2}+\frac{\alpha}{4}\left(x_{i}^{2}-1\right)^{2}-h \sum_{i} x_{i}\right],
$$

where $\alpha=2-A / J$ and $h=H \sqrt{B / \alpha J^{3}}$. Here the new energy scale is $F_{0}=\alpha J^{2} / B$, i.e., $\mathbf{F}=F / F_{0}$. The physical variable $x_{i}$ specifies the value of the the magnetic moment of the $i$-th particle oriented in the $z$ direction while the total magnetization of the system $M=\Sigma_{i} x_{i} \cdot{ }^{18-20}$ It is specifically interesting to investigate the situation when the particles form a ring or a loop geometry. Similar kind of loops were recently widely discussed as a candidate for magnetic memory elements. ${ }^{21}$ 
One important issue for the experimental investigation of artificial magnetic materials is the question about the creation of magnetic structures. What kind of magnetic structures can evolve due to temperature and field changes, due to the competition between the exchange energy and the anisotropy energy, and due to discreteness of the system? Depending on the number of particles $N$, how many distinct structures can be created? First let us consider the case when the external magnetic field is switched off, i.e., $h=0$. Stationary configurations of the Hamiltonian (4) are described by the $N$ nonlinear coupled equations

$$
-\left(x_{i-1}-2 x_{i}+x_{i+1}\right)+\alpha x_{i}\left(x_{i}^{2}-1\right)=0, i=1, \ldots, N
$$

with periodic boundary conditions. In the large $\alpha$ limit the system decouples and reduces to

$$
x_{i}\left(x_{i}^{2}-1\right)=0, i=1, \ldots, N .
$$

This set of equations has exactly $2^{N}$ stable solutions consisting of all possible "binary" strings $\mathbf{x}^{*}=\left(x_{1}^{*}, x_{2}^{*}, \ldots, x_{N}^{*}\right)$ with $x_{i}^{*} \in\{-1,1\}$. Provided that $\alpha$ is sufficiently large, the implicit function theorem then allows for the existence of solutions of Eq. (5) in terms of a power series expansion in $1 / \alpha$ about the asymptotic solution $\mathbf{x}^{*}$

$$
x_{i}=x_{i}^{*}+\frac{x_{i-1}^{*}-2 x_{i}^{*}+x_{i+1}^{*}}{2 \alpha}+\cdots .
$$

The first order correction term is proportional to $1 / \alpha$. However, due to internal symmetries within the strings the corresponding energies as well as the total magnetizations $M$ are highly degenerate. The number of nonequivalent strings can be determined by the action of the dihedral group $D_{N}$ consisting of $N$ translations $\left(x_{s} \rightarrow x_{s+i}\right)$ and $N$ mirror reflections $\left(x_{s+i} \rightarrow x_{s-i}\right)$ with respect to all symmetry axes. From the Redfield-Polya theory ${ }^{22}$ it can be shown that the number of nonequivalent strings increases exponentially as $(1 / N) 2^{N-1}$ with increasing $N$. These states are associated with kinks and antikinks related to the formation of domain walls.

Since two-dimensional maps allow representation of any stationary configuration of a Hamiltonian of the form (2) by a trajectory of a dynamical system, the solutions of our coupled set [Eq. (5)] are periodic orbits of the corresponding map equation. ${ }^{18,23}$ Due to our periodic boundary conditions there is neither chaotic behavior nor incommensurability involved.

\section{CLASSIFICATION OF POSSIBLE STRUCTURES}

Inserting the first order expansion of Eq. (7) into Eq. (4) yields the series expansion in $1 / \alpha$ for the free energy

$$
F=\sum_{i=1}^{N}\left(1-x_{i-1}^{*} x_{i}^{*}+\frac{x_{i}^{*}\left(4 x_{i-1}^{*}-x_{i-2}^{*}\right)-3}{2 \alpha}+\cdots\right) .
$$

Introducing the quantities $l$ and $l^{\prime}$ by $N-2 l:=\sum_{i=1}^{N} x_{i} x_{i-1}$ and $N-2 l^{\prime}:=\sum_{i=1}^{N} x_{i} x_{i-2}$, respectively, the total free energy per particle can be approximated by the simple expression

$$
F\left(l, l^{\prime}\right)=2 l-\frac{4 l-l^{\prime}}{\alpha} .
$$

Here, the quantity $l$ counts the sign changes between all nearest neighbors and $l^{\prime}$ those involving only next-nearest neighbors of the magnetic moments, respectively. The quantity $l$ specifies the number of kinks at the formation of domain walls which only appear in pairs. Note that this approximation is exact for the homogeneous ground states as well as for the two antiferromagnetic states. Moreover, in this approximation all configurations with the same number of domains have the same value of the energy. Within this approximation, when the magnetic field is nonzero the expansion, Eq. (7), is slightly modified to the form

$$
x_{i}=x_{i}^{*}+\frac{x_{i-1}^{*}-2 x_{i}^{*}+x_{i+1}^{*}}{2 \alpha}+\frac{h}{2 \alpha}+\cdots \text {. }
$$

Inserting this equation into the expression for a total magnetization we obtained very simple formula

$$
m(h)=\frac{1}{N} \sum_{i} x_{i}^{*}+\frac{h}{2 \alpha} .
$$

The homogeneous configurations $\mathbf{x}_{\mathbf{G}}{ }^{*}= \pm\{1,1, \ldots, 1,1\}$ with $l=l^{\prime}=0$ define the two degenerate ground states with $F_{G}=0$. The first excited state is specified by the simplest "magnetic one-soliton structure" generated by the symmetric configuration $\mathbf{x}_{\mathbf{S}}{ }^{*}=\{-1, \ldots,-1,+1,-1, \ldots,-1\}$ with $l=2$ and $l^{\prime}=2$ and having the energy $F_{S}=4-6 / \alpha+\cdots$. Such a soliton is similar to a stationary breather observed in many physical systems. ${ }^{24}$ The next excited state is represented by the pair kinks forming the domain wall structure generated by the asymptotic configuration $\mathbf{x}_{\mathbf{D}}{ }^{*}=\{-1, \ldots-1,-1,+1,+1, \ldots$, $+1,-1,-1,-1, \ldots\}$ with $l=2$ and $l^{\prime}=4$.

With an increasing number of particles the energy per particle of these specific structures including many sign reversals \pm 1 become arbitrarily close to the ground state and vanish in the large $N$ limit. Note that these configurations are locally stable. The total number of such locally stable configurations is equal to $2^{N}$. They are separated by a large barrier from the ground state. To illustrate the formation of such complicated energy landscape let us consider the case of two particles in detail. The dependence of the total energy on the values of magnetic moments $\left(x_{1}, x_{2}\right)$ for this system is presented in Fig. 1. From this figure we may see that there are four local minima which are well separated by four barriers. Each of these barriers is associated with a saddle point of the energy landscape. Two of these minima correspond to the ground state. The corresponding values of moments are equal to $\pm(1,1)$. The other two minima are metastable. The corresponding values of moments for these metastable minima are equal to $\pm(\sqrt{1-4 / \alpha},-\sqrt{1-4 / \alpha})$. Although all four minima are well separated in the space of individual magnetic moments, their separation in the energy space is very small, ie significantly smaller than the barrier heights associated with the saddle points (see, Fig. 1). Thus due to such an energy landscape the system could be locked in one of these minima with decreasing temperature, even if this minimum does not correspond to the ground state. ${ }^{25}$ 


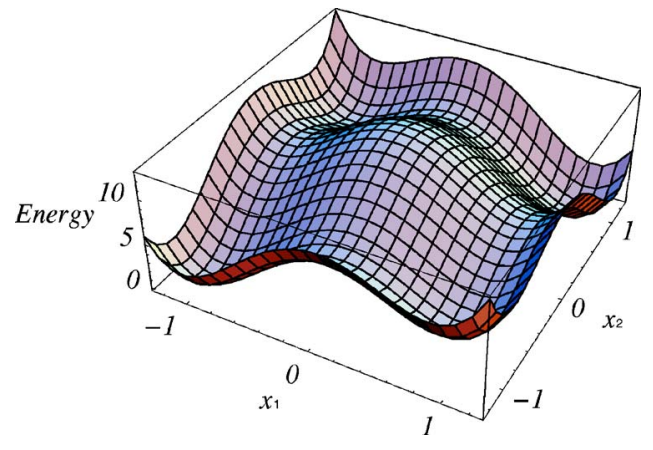

FIG. 1. (Color online) The energy landscape for the system consisting of two particles, i.e., the dependence of the total energy on the values of magnetic moments $\left(x_{1}, x_{2}\right)$. The picture illustrates that the difference between energy related to absolute minima arising at the values of moments $\pm(1,1)$ and the energy related to metastable minima arising at the points $\pm(\sqrt{1-4 / \alpha},-\sqrt{1-4 / \alpha})$ is much smaller than the barrier height defined by the saddle points presented on this figure.

Therefore, the formation of a particular structure will depend strongly on the history of the system, for example at which rate and from which temperatures and at which magnetic field the material was cooled or heated. The pair of domain walls created and described by the configuration $\mathbf{x}_{\mathbf{D}}^{*}$ has a higher energy than the soliton $\mathbf{x}_{\mathbf{S}}^{*}$. This indicates that there is a decoupling energy which is needed to decouple domains having different topological charges (kink from antikink). That is there is the energy cost in decoupling of the domain walls forming the soliton. In general domain walls are always created in pairs. One always needs the energy to perform a transition from the state with $l$ domain walls to the state with $l+2$ domain walls. Note that the highest energy $F=2(1-2 / \alpha) N$ is achieved for the antiferromagnetic configuration $x_{i}=(-1)^{i} \sqrt{1-4 / \alpha}$ with $l=N$ and $l^{\prime}=0$ or in other words for maximal number of domains. This structure exists only for $\alpha \geqslant 4$ and is physically stable only for $\alpha \geqslant 6$. Similarly, if the magnetization of one particle is zero $\left(x_{i}=0\right)$ the energy cost due to this "node" would be of the order of $\alpha$ $\gg 1$. The configurations with nodes are physically unstable saddle points and correspond to barriers separating the states with domain walls and solitons. With increasing and decreasing magnetic fields there arises multiple instability. At each such instability the saddle point associated with one of the barriers will coincide with the local minimum, and the locally stable configuration, which was associated with that minimum, ceases to exist. These metastable states and multiple instability created with increasing and decreasing magnetic field lead to a formation of multiple hysteresis loops, see below.

The classification of the metastable states is simple. The first is the two domain walls (bound state of kink + antikink). The next one consists of four domain walls (two kinks and two antikinks), etc. At zero magnetic field the energy difference between successive minima is of the order of 1 while the height of the barrier is always of the order of $\alpha \gg 1$. When the coupling constant $\alpha \gg 1$ we may expect that the system has some kind of glassy behavior where an ideal ferromagnetic ordering of the ferromagnetic particles is bro-
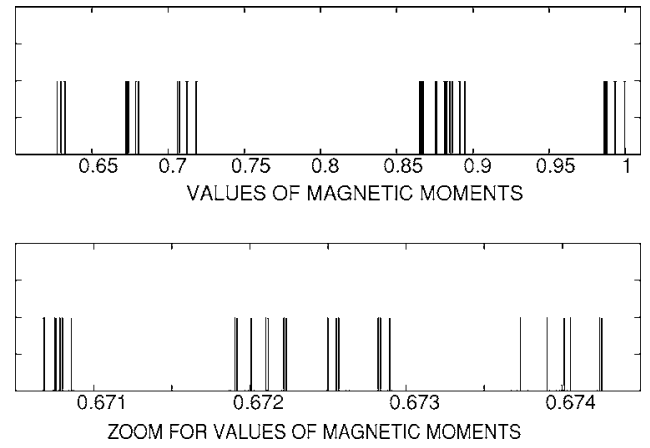

FIG. 2. A representative series of absolute values of magnetic moments of the individual particles are presented on the horizontal axes. The scale of the vertical axes is meaningless. Top: The distribution of magnetic moments for a chain consisting of $N=50000$ particles for the parameter $\alpha=8$. Bottom: The zoomed fine structure of the distribution of magnetic moments in the range of moment values from 0.6705 to 0.6745 . This distribution displays all features of the Cantor set. The total set of real values for magnetic moments is obtained from the set of absolute values presented in the figure with the use of the symmetry of Eq. (5), by simple substitution $x_{i}$ $\rightarrow-x_{i}$.

ken. The different $2^{N} / N$ locally stable distinct configurations of the domains may be analytically described up to arbitrary order with the parameter $\alpha \gg 1$. The distribution of magnetic moments and the energy spectrum will be presented below.

\section{DISTRIBUTION OF MAGNETIC MOMENTS}

The calculated values of the magnetic moments are shown in Fig. 2. Pronounced discrete structures appear marking forbidden regions. It is reminiscent of band chaos often found in nonlinear dynamical system theory. A physical origin of these bands and gaps may be related to a bound state of the stable topological excitations like kinks and antikinks. An example is the formation of a soliton. The fine structure is due to the kink-antikink interaction. This interaction modifies the value of the magnetic moment $x_{i}$ associated with each single $i$ th particle (see, Fig. 2).

The fine structures reveal some self-similarity reminiscent of those for the standard logistic map $x_{n+1} \rightarrow r x_{n}\left(1-x_{n}\right)$ with growth parameter $r$. When it is close to the critical constant $r_{c}=3.5699 \ldots$ it is specifying the onset of chaos, where the attractor is a Cantor set. In both cases the origin of the fractal structures is in the discreetness of the system and in the nonlinearity. In our system the latter is related to the interaction between domain walls. A crude estimate for the fractal dimension with the aid of a box counting algorithm gives the value $D=0.555$ compared to $D=0.538$ for the logistic map. ${ }^{26}$

In order to get an insight into the evolution of the fractal set, we calculate systematically all values of magnetic moments for all possible configurations. We consider small clusters (rings) with $N=1,3,5,7, \ldots$ and impose periodic boundary conditions $\left(x_{0}=x_{N}, x_{N+1}=x_{1}\right)$. For a single particle, $N=1$, we only have the two degenerate ground states. These two states correspond to the two values magnetic moments, $(x= \pm 1)$. For $N=3$ we find the two ground states and six soliton struc- 

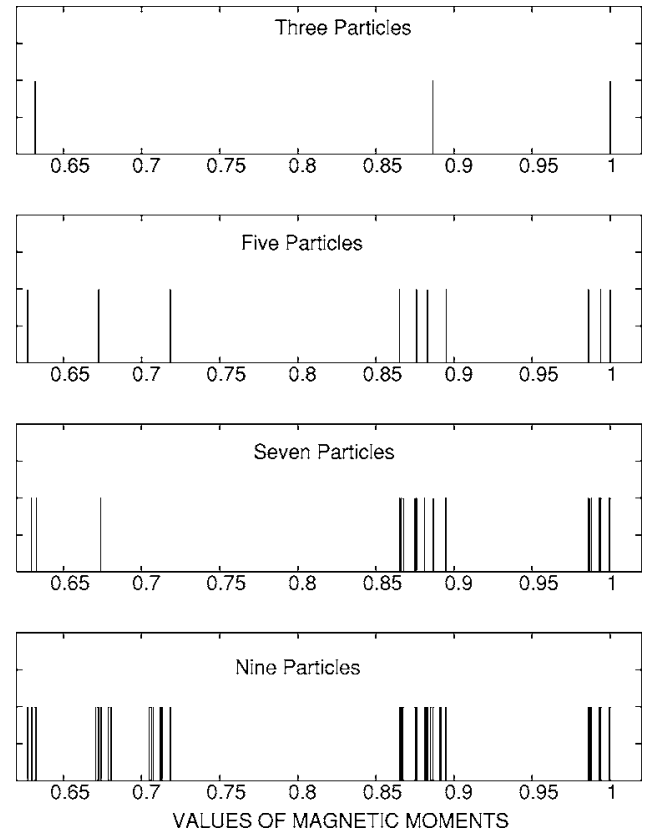

FIG. 3. The different absolute values of magnetic moments for all possible states (we call this set of lines as a distribution of magnetic moments) for a chain consisting of $N=3, N=5, N=7$, and $N=9$ particles, where the value of parameter $\alpha=8$. The total number of all possible locally stable states is equal to $2^{N}$. Already for the comparison of two cases of nine particles and seven particles we can see here the development of the first features of fractal formation: the fractal arises by appropriate triplication and quadruplication of spectral lines.

tures. Two ground states correspond to the homogeneous states: (111), (-1-1-1) and other six - to degenerate soliton states: $\quad(1,-1,1) ;(-1,1,1) ;(1,1,-1) ;(-1,1,-1) ;(-1,-1$, $1) ;(1,-1,-1)$. In Fig. 3 we present the different absolute values of magnetic moments, $\left|x_{i}\right|$, corresponding to all these different states. In particular, for $N=3$ and finite $\alpha$, the values of the magnetic moments for the asymptotic soliton state $(1,-1,1)$ are not equal to $1,-1,1$, respectively. The values of these magnetic moments can be calculated numerically and analytically from Eq. (5) provided that the parameter $\alpha$ is sufficiently large. For $\alpha=8$ the exact result for the asymptotic soliton state $(1,-1,1)$ is

$$
x_{1}=0.886, \quad x_{2}=-0.632, \quad x_{3}=0.886 \text {. }
$$

The absolute values of these numbers are presented and clearly seen in Fig. 3. These numbers together with the number 1.0 associated with homogeneous state are giving rise to three distinct values of moments depicted as three "spectral lines" in Fig. 3 (see the first box from the top). Thus, the two left of these three lines correspond to the single soliton structure associated with six soliton states while the right line corresponds to a homogeneous $(1,1,1)$ and $(-1,-1,-1)$ states. Let us denote this set of three lines as $T$, i.e., $S_{3}=T_{3}$ and call it a triplet. The index indicates the number of particles at which this triplet cluster arises. Then, when the number of particles $N$ increases by 2 and becomes equal to $N$ $=5$, each line from the set $T_{3}$ is split. Then there arise new clusters of spectral lines. There, each of two outer lines from the $T_{3}$ cluster will be split into a new $T_{5}$ cluster (see, top of Fig. 3). Therefore, the number of $T$-type clusters will be increased by a factor of 2 ,

$$
T_{5}=2 T_{3} .
$$

On the other hand, the central line from the $T_{3}$ cluster will be split into a new set consisting of four lines. Let us denote this set of four lines as $Q$ and call it as quadruplet. Here each quadruplet $Q$ is surrounded by two triplets. The total number of quadruplet clusters arising for the number of particles $N$ $=3$ is equal to $Q_{3}=0$, (see, top of Fig. 3). The number of the quadruplet clusters arising in the case of $N=5$ is equal to

$$
Q_{5}=T_{3}+4 Q_{3} \text {. }
$$

Obviously, $Q_{5}=1$ (see, the second figure from the top of Fig. 3 ). The total set of spectral lines for $N=5$ particles $S_{5}$ will consist of two triplet sets $T_{5}$ and one quadruplet set $Q_{5}$

$$
S_{5}=2 T_{5}+Q_{5} \text {. }
$$

Thus, with increasing number of particles from $N=3$ to $N$ $=5$ particles the number of spectral lines will be increased and, according to Eq. (10), it will be equal to 10 (see, the second box from the top of Fig. 3).

At the next step of increasing number of particles from $N=5$ to $N=7$ particles, the number of spectral lines will be further increased. All these lines will be grouped into triplet and quadruplet clusters. Again, here each triplet cluster will give rise to two new triplet clusters $T_{7}=2 T_{5}$ and to one quadruplet cluster. Also each quadruplet cluster will give rise to four quadruplet clusters. In total there will arise $Q_{7}=4 Q_{5}$ $+T_{5}$ quadruplet clusters (see, the third figure from the top of Fig. 3). In general in each case when the number of particles will be increased by 2 , the number of triplet clusters will be doubled

$$
T_{2 n+1}=2 T_{2 n-1} \text {. }
$$

Equation (16) allows us to calculate explicitly the number of lines grouped into triplet clusters $N_{T_{2 n+1}}=2^{n}$. On the other hand, the number of quadruplet clusters will be described by the recursion relation

$$
Q_{2 n+1}=T_{2 n-1}+4 Q_{2 n-1} .
$$

One can easily calculate the total number of lines $N_{Q}$ grouped into quadruplet clusters, i.e.,

$$
N_{Q_{2 n+1}}=\frac{1}{2}\left(2^{2 n}-2^{n}\right) \text {. }
$$

Applying successively these rules one can reproduce the set of values of magnetic moments for a system, having any number of particles. The splitting of spectral lines and their grouping into the triplet and quadruplet clusters may be nicely illustrated with the diagram presented in Fig. 4.

Each triangle corresponds to a triplet cluster or to three spectral lines. Each square corresponds to a quadruplet cluster or to four values of magnetic moments (to four spectral lines). With increasing number of particles a quadruplet cluster (a square) is split into four quadruplet clusters (four squares) and a triplet cluster (a triangle) is split into one 


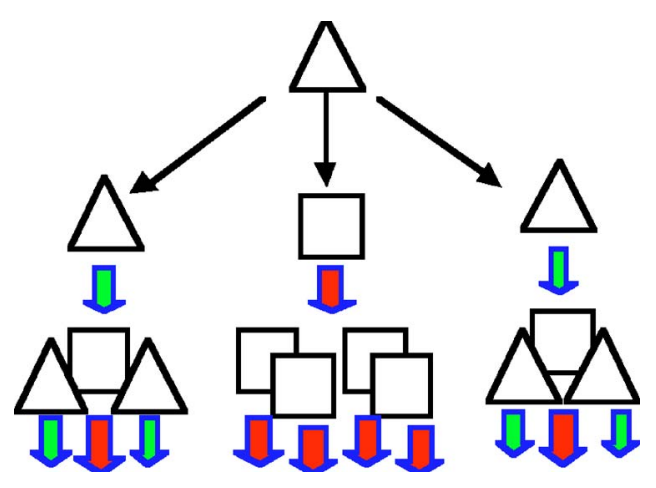

FIG. 4. (Color online) The schematic diagram presented for a description of the clusterization of the values of magnetic moments into triplet and quadruplet clusters. This clusterization also describes the evolution of the fractal structure when the number of particles in the chain increases. Here, one can see how the fractal magnetic structure evolves when we are moving from above, for $N=3$ (the triplet cluster or three spectral lines), $N=5$ (two triplets and one quadruplet clusters corresponding to ten spectral lines in total), and $N=7$ particles (four triplets and six quadruplets corresponding to 36 spectral lines in total), respectively.

quadruplet (one square) and two triplet states (two triangles). The total number of distinct values of the magnetic moments increases according to the exponential law $S_{2 n+1}=T_{2 n+1}$ $+Q_{2 n+1}$ equal to

$$
S_{2 n+1}=2^{2 n-1}+2^{n-1} .
$$

For an even number one can show that the increase is

$$
S_{2 n}=2 S_{2 n-1} .
$$

To summarize here, each time with increasing number of particles the spectral lines appear there in triplets and quadruplets. Further on, each triplet splits into one quadruplet and two triplets. Each quadruplet will split into a new quadruplet, i.e., each spectral line from the quadruplet set splits into four other lines, which in the next round, with an increased number of particles, will create quadruplets. As a result, this cluster multiplication will lead to formation of multifractal structures. This multifractal spatial distribution of the magnetic moments described above is difficult to access experimentally, although modern techniques, like scanning tunneling microscopy (STM) and atomic force microscopy (AFM) as well as Kerr rotation may be able to fulfil such a task. With such experiments one can observe the spectral lines from the fractal associated with the one or a few such states. It will be probably very difficult to see all spetral lines associated with all different states of this ensemble although some fraction of them can be observed.

\section{THE ENERGY SPECTRUM}

Due to the Cantor set structure of the distribution of the values of magnetic moments also the energy spectrum is also expected to be fractal. Indeed, the different energy values arise from interaction between topological defects (solitons and domain walls). These values also form a Cantor structure. The appearance of these fractal structures apparently
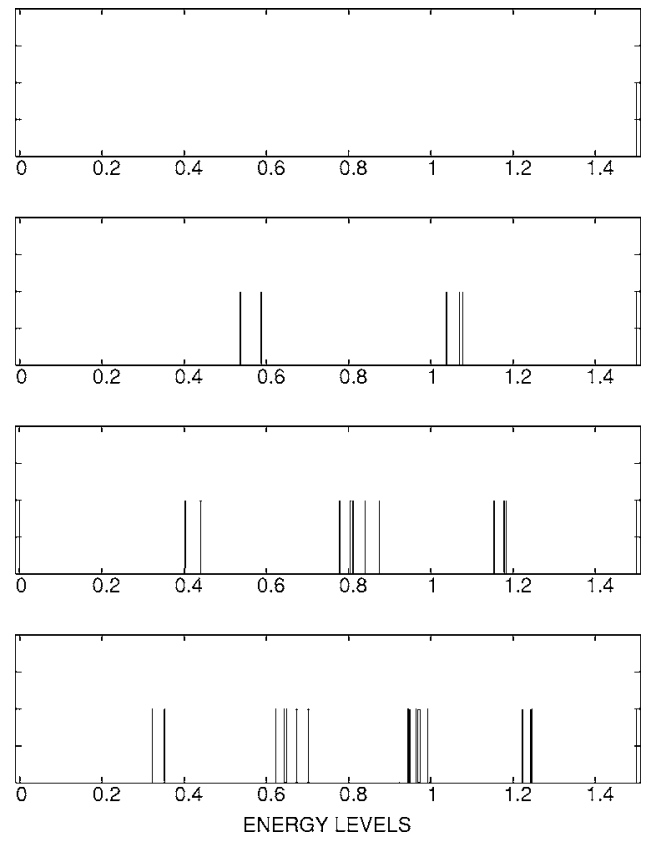

FIG. 5. On the horizontal axes we present the value of the energy for all possible domain configurations of the chain of magnetic particles. This distribution of energy values forms the energy spectrum. In the figure we present the energy spectrum for a chain consisting of (from above) $N=4, N=6, N=8$, and $N=10$ particles, where the parameter $\alpha=8$. Already for ten particles (the bottom figure) we can see here the first features of the fractal formation in the energy spectrum.

stems from a fundamental reason related both to the discrete nature of the system consisting of small magnetic particles and to the large but finite number of weekly interacting topological excitations. Figure 5 clearly shows that with increasing number of particles more fine energy structure appears. In this figure the ground state with $E=0$ corresponds to the left boundary spectral line. The antiferromagnetic state with the highest energy per particle $E / N=2(1-2 / \alpha)$ located on the right boundary of the energy spectrum.

We observe that with increasing number of particles the fractal develops in terms of grouping into clusters whose number increases with increasing number of particles. Already at ten particles we may clearly separate each level of the fractal. The most important novel result is the energy landscape, which consists of exponentially many locally stable minima separated by large barriers represented by unstable saddle points. Each of these minima corresponds to the state with some fixed number of sign reversals specifying the domain structure. Even if such a number is fixed the states associated with different configurations or rearrangement of these domains will correspond to different or the same degenerate minima. These energy levels, whose number is exponentially increasing, do not differ much from each other; however, the barrier height between the corresponding minima increases with increasing values of $\alpha$. Since all these configurations are locally stable and are separated from each other by large barriers we may conclude that some kind of a glassy state should arise here. ${ }^{25,27}$

The landscape structure will be reflected in the measured quantities mainly on the magnetization measurements where 
only one minimum can be revealed at the time. Each of the local minima will correspond to a different value of the magnetization. Of course to reveal the fractal features one has to perform high resolution measurements.

\section{TOTAL MAGNETIZATIONS AND HYSTERESIS}

It is clear that the external magnetic field will have a strong influence on the physical properties of a chain made of these magnetic particles. Let us apply the field along the main axes of symmetry, i.e., oriented in the $z$ direction. With increasing magnetic field the complicated energy landscape associated with domain structure changes. Initially the degeneracy between different local minima will be lifted. Some minima become deeper and some minima become shallow and with further increasing field some minima start to disappear. With next increasing field more and more minima will disappear.

With such disappearance of some minima the corresponding values of the magnetic moments will disappear and more and more moments will be completely polarized. That is depending on the direction of the magnetic field, they will take their maximal possible value. With increasing polarization of some moments the associated fractal set will have fewer points. For sufficiently large magnetic field all moments will be polarized. At the field higher than this critical field the complicated energy landscape will disappear and will be substituted by one big absolute minimum associated with the fully polarized state. The fractal features of the structure will disappear as well.

Thus the fractal structure (or the fractal dimension) is field sensitive. Because the total magnetization is a sum of the magnetic moments of individual particles, the changing of the fractal structure of the magnetic moments is reflected in the behavior of the total magnetization in a magnetic field. First of all because the total set of magnetic moments associated with individual particles forms the fractal we expect that the total magnetization, to which each moment is contributing, will also form some fractal. This has been verified for the distribution of the values for total magnetization of the chain at zero field and we have found that this set also has fractal features.

The total magnetization as a function of the external magnetic field are presented in Fig. 6. The figure reveals that practically at each fixed value of magnetic field this dependence is multivalued and represents some fractal features very sensitive to magnetic field. From this figure one may also see that this distribution consists of a few patches or clusters of points. In fact each of these patches or branches corresponds to a fixed even number of domains created in the chain. The uppermost and lowest branches presented in this figure correspond to fully polarized states and describe effectively the formation of the conventional hysteresis loop of a ferromagnet made of these particles (see the dashed lines on the Fig. 6). The second uppermost (or the second lowest) branch corresponds to a single soliton which is formed as a bound state of the two nearest domain walls. Probably such soliton branches of the hysteresis are very generic to arbitrary linear chains made of small magnetic particles. Other

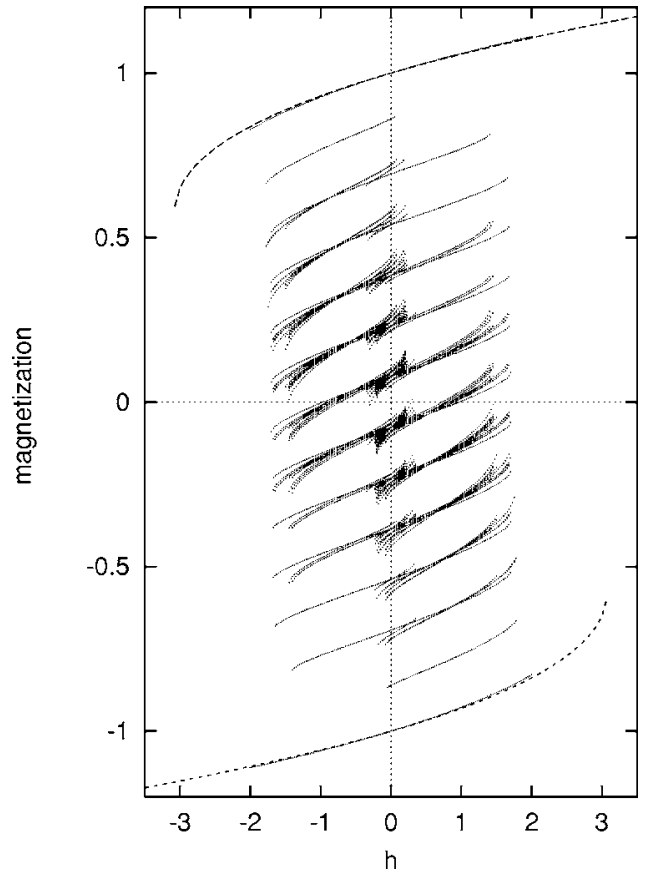

FIG. 6. All possible values of the total magnetizations $\mathbf{M}$ (the vertical axes) as a function of the magnetic field $h$ (the horizontal axes) in arbitrary units for the value of the parameter $\alpha=8$. The magnetic field is changing in steps $\Delta h=0.025$. The magnetization (its values are associated with points in the figure) is a multivalued function of the external magnetic field. The different values of the magnetization are related to all possible domain structures formed in the chain consisting of 13 particles. The dashed lines correspond to fully polarized states and present the form of the conventional hysteresis loop of a ferromagnet.

branches or clusters of the distribution in magnetization have nontrivial fractal features. From Fig. 6 one also sees that with increasing or decreasing magnetic field the fractal-like structure of the total magnetization becomes less and less dense. This is in complete agreement with the discussion presented above.

One may also see from Fig. 6 that the total distribution of the magnetization is changing in a nonmonotonic way with the field. At zero magnetic field we know that this distribution is associated with the maximum number of $2^{N}$ different locally stable partially degenerate minima. However, when we slightly increase or decrease the magnetic field the symmetry $x_{i} \rightarrow-x_{i}$ in Eq. (5) is broken such that the degeneracy becomes smaller. Then each patch or a cluster in this distribution looks denser and even broader compared with the case of zero magnetic field. Though for small magnetic fields all $2^{N}$ local minima still exist, their number will inevitably decrease with increasing magnitude of the magnetic field.

There is a one to one correspondence between each minimum of the energy landscape and a specific domain configuration. Such domain configurations are very sensitive to magnetic field and change with the field by a very nontrivial way. For example, when the field is equal to $h= \pm 0.5$ the size or the width of each branch of the total distribution of magnetization set becomes very small (see Fig. 6) (except two uppermost and two lowest branches which were already 
small). At this fixed value of the field the number of the clusters remains the same as at zero field. It is exactly equal to the number of states with different even number of domains. This shrinking of the branch patches at such magnetic field is related to the effect that some minima associated with some domain (or precisely speaking, with soliton+domain) configurations have been disappeared already. But with the next increasing magnetic field, at $h=0.75$ and at $h=1.0$ we see from the Fig. 6 that the size of the clusters is growing again. This cluster growth is presumably related to an increase of the distance between local minima and corresponding energies of the complicated energy landscape. With further increasing field, some of the minima disappear and the energy landscape becomes less and less complicated. As the result the clusters become less and less dense.

Finally at the critical field $h_{c} \approx 1.75$ the remaining chunks of the fractal structure (see Fig. 6) vanish. At fields larger than $\left|h_{c}\right|$ all cluster features of the magnetization distribution have disappeared. There remains only two local minima associated with fully polarized state oriented along or opposite to the external field (see, the dashed lines in Fig. 6).

The existence of such complicated energy landscape indicates that the magnetic properties of the chain of small particles will be very different from conventional bulk magnets. In particular, the magnetization hysteresis loop may be very nontrivial. Indeed, the fractal structure presented on the Fig. 6 also suggests that a large variety of different hysteresis loops are theoretically possible. In particular, the magnetization $\mathbf{M}$ as a function of the field $h$ is not necessarily smooth but can increase in steps. This fundamental mechanism giving rise to a series of minute jumps in the magnetization is the so-called Barkhausen effect ${ }^{28,29}$ which gave first experimental evidence of these magnetic instabilities. Thus the fact that all possible values of the total magnetization form a fractal leads to the result that the fractal hysteresis loop can be constructed from the Cantor set of the values of the total magnetization.

Note that a specific domain structure corresponds to the system being trapped in one of these local energy minima. However, because it is a metastable state a slight change of the strength of the applied field $H$ can easily destabilize a specific domain structure. The less stable structures are domain wall configurations forming the soliton structure where two domain and antidomain walls are creating a bound state. It is sufficient that with increasing field the local minimum of the energy landscape is transformed into a saddle point and such domain configuration disappear.

The system then evolves toward to a situation when some other metastable configuration will disappear and so on. These rearrangements can be quite localized in space or may involve even the whole domain structure.

Provided that the magnetic field is not too strong, the distribution of the total magnetizations $\mathbf{M}$ at each fixed value of the magnetic field are also reminiscent of Cantor-set structures (see Fig. 6). Obviously, there will be a critical field value, where the fractal structure disappears. It is also important to note that the set of the hysteresis curves is different for the case of the chain with even and odd numbers of particles. For example, the chain consisting of an odd number of particles will never be demagnetized, i.e., to have the

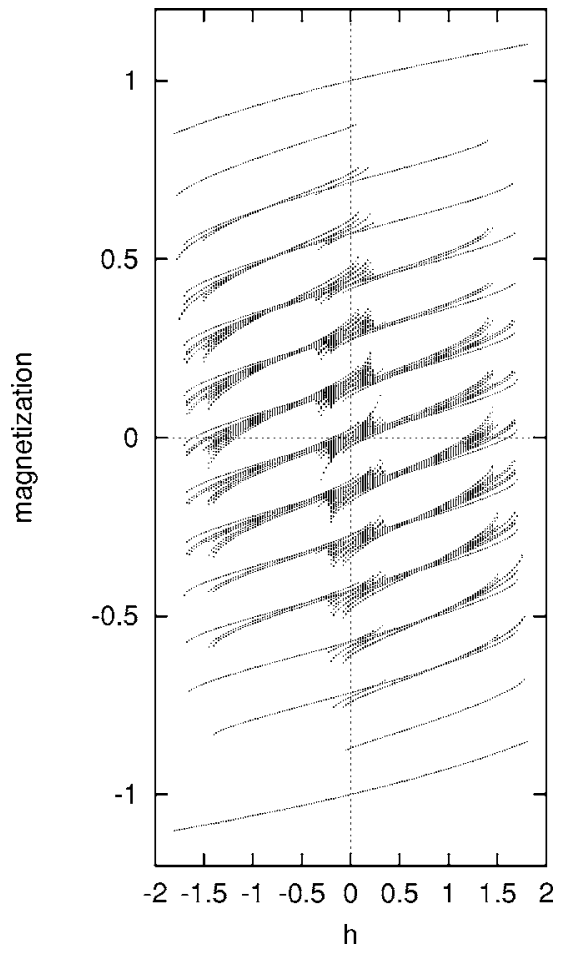

FIG. 7. The notations are the same as in Fig. 6 but for the chain consisting of an even number of particles, $N=14$.

same number of positively and negatively oriented magnetic moments. In other words, there are no values of magnetization in the vicinity of the origin, see Fig. 6. The situation is completely different when the chain consists of an even number of particles. Then the chain can be completely demagnetized and there is a subset of the values of magnetization distributed in the vicinity of zero, see Fig. 7. Such a completely demagnetized state of the chain may correspond to a single domain configuration. Generally speaking, in such a demagnetized configuration the number of domains may be different from one. However, the number of positively and negatively oriented magnetic moments must be always equal to each other. This can obviously be satisfied for the chain having an even number of particles but is impossible for the chain having an odd number of particles (see, for comparison, the branches of the hysteresis loop obtained for the cases of the chain with an even and odd number of particles presented in Figs. 6 and 7, respectively).

\section{POSSIBLE EXPERIMENTS FOR A RECOVERING OF BARKHAUSEN JUMPS}

The fractal studies in the distribution of the total magnetization presented in Figs. 6 and 7 may be detected in experiments with the use of the fast cooling rates at different field strengths. At fast cooling the configuration formed at high temperatures will be associated with one of the metastable states and therefore at low temperatures it will be frozen and corresponds to one specific value of the fractal presented in Fig. 6 and 7. Since at high temperatures $(\alpha \rightarrow 0)$ the energy landscape is very shallow, the high temperature state does 
not correspond to a specific deep minimum. Then all types of thermal fluctuations exist and all types of configurations will be created. With fast cooling these configurations could be frozen into one of the configurations of the multivalley energy landscape, which will be revealed by the fractal structure of the measured values of the total magnetization.

The complicated structure of the hysteresis loop presented in Figs. 6 and 7 may be observed in a series of the field cooled and zero field cooled experiments. Let us note that this structure is a result of the complicated energy landscape consisting of the many locally stable minima separated by large barriers. The formation of these minima and the barriers is beginning with decreasing temperature starting from the critical temperature $T_{c}$ of the bulk ferromagnetic transition. With the next decreasing temperature the minima become deeper and deeper while the barrier height increases. At all temperatures the energy positions of the bottom of the minima are not very different from each other. Such difference is determined by the weak dipole-dipole interaction between particles which is characterized by the coupling constant $J$. Therefore, with decreasing temperature, when $T_{c}$ $-T \gg J$, we will have a system where there are many, practically equivalent, minima separated by large barriers. A simple estimation gives that for a particle consisting of $10^{3}$ magnetic atoms the value of $J$ must be significantly smaller than $10 \mathrm{~K}$. The latter, of course, depends on the distance between particles $r$ and ceases as $1 / r^{3}$. Therefore, it is clear that the condition when $T_{c}-T \gg J$ can be easily realized. Obviously that when the temperature decreases very fast the system may be easily trapped in any one of these minima. In which of these minima the trapping will occur does depend on the cooling rate and on initial configuration created by a thermal fluctuation as well as on the strength of the applied magnetic field. This property of the system may serve as a basis of the following experiments.

First, if we cool the system in a strong magnetic field and then continuously change the field $h$ we will recover the main features of the bulk hysteresis loop (see the dashed lines in Fig. 6). In this case the system will be trapped in a fully ferromagnetic state associated with the absolute minimum. Moreover, the system will even be trapped in this state when the minimum becomes not absolute, which happens with the next continuous change of the field $h$. However, if we cool the system in a weak or zero field the resulting minimum in which the system will be trapped will depend on the cooling rate and on the initial high temperature state. With the very slow cooling rate the system will have time to termalize into the bottom of absolute minimum. However, due to the barriers of much height that surround these minima, when the cooling rate is not slow the system will be trapped in other locally stable minima, which is different from the absolute mininum and has different values of the total magnetization. With the very fast cooling rate in a weak or zero field the system may be trapped in any minima having locally stable configurations and characterized by different values of magnetization. Such a dramatic difference between the field cooled and zero field cooled magnetizations reminds us of the situation that is usually arising in spin glasses.

Then, when the system is trapped in one of these locally stable minima, the continuous change of the field $h$ may induce the transition of the system from this minimum into another neighboring local minimum that has the closest magnetic configuration. Such a transition must occur when a certain branch of the hysteresis set presented in Figs. 6 and 7 is terminated and such transition will be reflected in the Barkhausen jump of the total magnetization $M$. This will be seen in the experiment measuring the total magnetization of the system. With the next continuous change of the field $h$, first the magnetization will be changed continuously until this branch of the hysteresis set will be terminated. Then another transition into a new minimum and another Barkhausen jump in the total magnetization will occur. Thus if the system will be trapped originally in one of these locally stable (not absolute) minima, due to the fast cooling or some other way, then with continuous change of the field $h$ we will recover a series of Barkhausen jumps in the total magnetization $M$. Such a Barkhausen (devil) staircase may be different from one experiment to another, from one measurement to another reflecting a very rich and complicated energy landscape associated with the system consisting of these small particles.

\section{SUMMARY}

Thus, our studies led us to amazing results, namely the spatial structures of domains in a chain made of small magnetic particles show fractal self-similarity. Such a spatial distribution of magnetic moments associated with different chains or clusters of particles is a difficult task to measure on experiments, although modern techniques, such as STM and AFM as well as the Kerr rotation, may allow such a task. However, from our point of view, the most important result obtained is the fractal structure of the energy spectrum. The energy landscape, which gives rise to such a fractal spectrum, is associated with the creation of domains and fractal values of the total magnetization. This landscape energy surface consists of many locally stable minima separated by large barriers. Each of these minima corresponds to the state with some fixed number of domains. Even if such a number is fixed the states associated with different configurations or rearrangement of these domains will correspond to different or the same minima. This is precisely the situation arising in a glassy system. The shape of the energy landscape leads us to the conclusion that the system formed from magnetic particles is some kind of magnetic glass associated with the creation of domains (see also Ref. 27). We propose to make a detailed experimental investigation of systems made of small magnetic particles (chains or clusters) to identify this glassy character and the fractal features of their domain structure. In this respect it might be useful to measure the magnetization at zero field as well as in cooled regimes as commonly practiced in experiments on spin glasses. Due to these energy landscapes described above the corresponding magnetic structures at very low temperatures are very stable with respect to thermal as well as to quantum fluctuations. To reveal these fractals the experiments associated with fast cooling should be setup. The repetition of the fast cooling from high temperatures may drive the system to settle in a different valley of the energy landscape. Measurement of the 
total magnetization at each lap of cooling with the same or different cooling rates may provide a set of numbers reminiscent of parts of a fractal. The latter will depend on the shape and the number of particles of which the nanostructure is formed. Since different clusters will be associated with different fractals, then in general these studies may lead to the development of a type of spectroscopy where, with the aid of fast cooling magnetization measurements, the structure of small clusters may be identified. These findings could also open areas of applications for devices having very controllable and stable giant magnetoresistance properties. ${ }^{28}$

There is another interesting example of the systems, where the described fractal structures can arise as well. ${ }^{30}$ These are one-dimensional chains and two-dimensional arrays of $\pi$ rings. ${ }^{31,32}$ A single $\pi$ ring is a superconducting loop consisting of Josephson junctions where there is at least one $\pi$ junction. ${ }^{33}$ The phase shift by $\pi$ in such a junction results in the formation of an orbital moment on the ring (see for details Ref. 33). Such orbital moments give rise to a paramagnetic Meissner effect ${ }^{33}$ observed in cuprate superconductors. ${ }^{34,35} \mathrm{~A}$ chain or a planar array of electrically isolated $\pi$ rings could be treated as a set of magnetic moments oriented perpendicular to the plane and interacting via magnetic dipole forces. This dipole-dipole interaction modifies the values of the orbital magnetic moments and leads to the formation of the fractal in the way decribed in this paper.

\section{ACKNOWLEDGMENTS}

We are grateful to F. Aliev, J. W. Clark, D. Edwards, G. Gehring, C. Krattenthaler, and John Samson for useful discussions. The work has been supported by European Science Foundation in the framework of the network program: Arrays of Quantum Dots and Josephson Junctions as well as the EPSRC Grant No. GR/S05052/01.
${ }^{1}$ M. A. Howson, Contemp. Phys. 35, (5) 347 (1994).

${ }^{2}$ C. Binns, Surf. Sci. Rep. 44, 1 (2001).

${ }^{3}$ C. Binns, S. Louch, S. H. Baker, K. W. Edmonds, M. J. Maher, and S. E. Thornton, IEEE Trans. Magn. 38, 141 (2002).

${ }^{4}$ H. Ardhuin, J. N. Chapman, P. R. Aitchison, M. F. Gillies, K. J. Kirk, and C. D. W. Wilkinson, J. Appl. Phys. 88, 2760, (2000).

${ }^{5}$ W. Schepper, A. Hütten, and G. Reiss, J. Appl. Phys. 88, 993 (2000).

${ }^{6}$ M. V. Gvozdikova and A. S. Kovalev, Low Temp. Phys. 25, 1295 (1999).

${ }^{7}$ F. V. Kusmartsev, H. S. Dhillon, and M. D. Crapper, J. Magn. Magn. Mater. 198-199, 743 (1999).

${ }^{8}$ A. Moser, K. Takano, D. T. Margulies, M. Albrecht, Y. Sonobe, Y. Ikeda, S. Sun, and E. Fullerton, J. Phys. D 35, R157 (2002).

${ }^{9}$ S. P. Parkin, C. Kaiser, A. Panchula, P. Rice, M. Samant, and S.-H. Yang, Nat. Mater. 3, 862 (2004).

${ }^{10}$ S. P. Parkin, X. Jiang, C. Kaiser, A. Panchula, K. Roche, and M. Samant, Proc. IEEE 91, 661 (2003).

${ }^{11}$ D. R. Lee, J. W. Freeland, G. Srajer, V. Metlushko, cond-mat/ 0309672 (unpublished).

${ }^{12}$ R. P. Cowburn, J. Phys. D 33, R1 (2000)

${ }^{13}$ J. Rothman, M. Klaui, L. Lopez-Diaz, C. A. F. Vaz, A. Bleloch, J. A. C. Bland, Z. Cui, and R. Speaks, Phys. Rev. Lett. 86, 1098 (2001).

${ }^{14}$ R. P. Cowburn, D. K. Koltsov, A. O. Adeyeye, M. E. Welland, and D. M. Tricker, Phys. Rev. Lett. 83, 1042 (1999).

${ }^{15}$ R. P. Cowburn, A. O. Adeyeye, and M. E. Welland, Phys. Rev. Lett. 81, 5414 (1998).

${ }^{16}$ R. P. Cowburn and M. E. Welland, Science 287, 1466 (2000).

${ }^{17}$ R. P. Cowburn, Phys. Rev. B 65, 092409 (2002)

${ }^{18}$ F. V. Kusmartsev and K. E. Kürten, in Lecture Notes in Physics, edited by J. W. Clark and M. L. Ristig (Springer-Verlag, NY, 1997), Vol. 284

${ }^{19}$ H. S. Dhillon, F. V. Kusmartsev, and K. E. Kürten, J. Nonlinear
Math. Phys. 8, 38 (2001).

${ }^{20}$ K. E. Kürten, in Condensed Matter Theories, (Nova Science, New York, 2000) Vols. 14 and 15.

${ }^{21}$ P. Vavassori, M. Grimsditch, V. Novosad, V. Metlushko, and B. Illic, Phys. Rev. B 67, 134429 (2003).

${ }^{22}$ K. E. Kürten and C. Krattenthaler, in Condensed Matter Theories, (Nova Science New York, 2004) Vol. 18.

${ }^{23}$ P. Bak and V. L. Pokrovsky, Phys. Rev. Lett. 47, 958 (1981).

${ }^{24}$ D. K. Campbell, S. Flach, and Y. S. Kivshar, Phys. Today 57, 43 (2004).

${ }^{25}$ D. Feinberg and F. V. Kusmartsev, Physics in Local Lattice Distortions, edited by Oyanagi and A. Bianconi (AIP, New York, 2001), p. 262.

${ }^{26}$ P. Grassberger, J. Stat. Phys. 26, 173 (1981)

${ }^{27}$ P. E. Jönsson, S. Felton, P. Svedlindh, P. Nordblad, and M. F. Hansen, Phys. Rev. B 64, 212402 (2001).

${ }^{28}$ P. Grünberg, Phys. Today 54, 31 (2001).

${ }^{29}$ H. Barkhausen, Z. Phys. 20, 401 (1919).

${ }^{30}$ F. V. Kusmartsev, D. M. Forrester, and M. S. Garelli, in Physics of Superconducting Phase Shift Devices, edited by A. Barone, E. Sarnelli, F. Tafuri and G. Testa (Ischia, Napoli 2005) p. 21.

${ }^{31}$ H. Hilgenkamp, H.-J. Ariando, H. Smilde, D. H. A. Blank, G. Rijnders, H. Rogalla, J. R. Kirtley, and C. C. Tsuei, Nature (London) 422, 50 (2003).

${ }^{32}$ J. R. Kirtley, C. C. Tsuei, Ariando, H.-J. H. Smilde, and H. Hilgenkamp, cond-mat/0503236 (unpublished).

${ }^{33}$ F. V. Kusmartsev, Phys. Rev. Lett. 69, 2268, (1992).

${ }^{34}$ P. Svedlindh, K. Niskanen, P. Norling, P. Nordblad, L. Lundgren, B. Lönnberg, and T. Lundström, Physica C 162-164, 1365 (1989).

${ }^{35}$ W. Braunisch, N. Knauf, V. Kataev, S. Neuhausen, A. Grütz, A. Kock, B. Roden, D. Khomskii, and D. Wohlleben, Phys. Rev. Lett. 68, 1908 (1992). 